\title{
Switching speed distribution of spin-torque-induced magnetic reversal
}

\author{
J. $\mathrm{He}^{a}$, J. Z. $\mathrm{Sun}^{b}$ and S. Zhang ${ }^{a}$ \\ a) Department of Physics and Astronomy, \\ University of Missouri-Columbia, Columbia, MO 65211 \\ b) IBM T. J. Watson Research Center, \\ P. O. Box 218, Yorktown Heights, NY 10598.
}

\begin{abstract}
The switching probability of a single-domain ferromagnet under spin-current excitation is evaluated using the Fokker-Planck equation(FPE). In the case of uniaxial anisotropy, the FPE reduces to an ordinary differential equation in which the lowest eigenvalue $\lambda_{1}$ determines the slowest switching events. We have calculated $\lambda_{1}$ by using both analytical and numerical methods. It is found that the previous model based on thermally distributed initial magnetization states [1] can be accurately justified in some useful limiting conditions.
\end{abstract}




\section{INTRODUCTION}

Fast and reliable nanosecond level writing is essential for spin-torque-induced switching in memory and recording technologies. One intrinsic source for write threshold distribution is thermal fluctuation, two aspects of which could affect reversal time $\tau_{s}$. First, the initial position of the magnetic moment is thermally distributed at the time the reversal field or current is applied, causing a variation in switching time. Secondly, during reversal, thermal fluctuation would modify the orbit, causing additional fluctuation for $\tau_{s}$ even for identical initial conditions. A reversal time distribution due to a thermalized initial condition was

recently estimated [1]. Here we present a Fokker-Planck formulation for the full problem including both thermalized initial condition and reversal orbit with estimates for the reversal time and its distribution.

Fokker-Planck equations have been successfully applied to macro-spin systems with a static thermal distribution for the extraction of a small thermal escape probability. For example, the distribution for a thermally activated reversal has been analytically and numerically obtained when the applied magnetic field is smaller than the coercive field [2, 3, 4, 5, 6]. There the switching is through thermally assisted transition from one metastable energy minimum to equilibrium. The ensemble-averaged $\tau_{s}$ depends exponentially on the ratio between the reversal energy barrier and thermal energy $k_{B} T$. These subthreshold results have recently been extended to include the effect of spin-torque, which modifies the effective temperature [7, 8, 9].

For fast, dynamically driven switching, the applied spin-torque is above the zerotemperature reversal threshold. For finite temperature, this leads to a time-dependent Fokker-Planck equation in which the time-evolution of the probability represents the thermalized reversal of the macro-spin. Here we solve this problem in the colinear geometry with a uniaxial anisotropy energy landscape and the presence of a spin torque.

\section{FOKKER-PLANCK EQUATION}

Consider an ensemble time-dependent magnetization probability density $P\left(\mathbf{n}_{\mathbf{m}}, t\right)$, where $\mathbf{n}_{\mathbf{m}}$ is the unit vector describing the direction of the macro-spin moment $\mathbf{m}$; in spherical coordinates $\mathbf{n}_{\mathbf{m}}$ is characterized by $(\theta, \phi)$. Before one turns on the magnetic field and the 
current, the probability density takes its equilibrium value. For a uniaxial anisotropy-only situation,

$$
P\left(\mathbf{n}_{\mathbf{m}}, 0\right)=P_{0} \exp \left(-\xi \sin ^{2} \theta\right)
$$

for $0 \leq \theta \leq \pi / 2$ and $P\left(\mathbf{n}_{\mathbf{m}}, 0\right)=0$ for $\pi / 2<\theta \leq \pi$, where $P_{0}$ is the normalization factor $\left(\int_{0}^{\pi} P \sin \theta d \theta=1\right), P_{0} \approx 2 \xi$, if we consider $\xi \equiv K V / k_{B} T \gg 1$ where $K$ is the anisotropy constant and $V$ is the volume of nanomagnet. To determine $P\left(\mathbf{n}_{\mathbf{m}}, t\right)$ after one turns on the field and the current at $t=0$, we need to solve the Fokker-Planck equation given below

$$
\frac{\partial P}{\partial t}+\nabla \cdot \mathbf{J}-D \nabla^{2} P=0
$$

where $\mathbf{J}$ is the probability current and $D=\alpha \gamma k_{B} T / m$ is the diffusion constant, $\alpha$ is the damping parameter, $\gamma$ is the gyromagnetic coefficient, and $m=|\mathbf{m}|$. The probability current is

$$
\mathbf{J}=P \frac{d \mathbf{n}_{\mathbf{m}}}{d t}=-\gamma P \mathbf{n}_{\mathbf{m}} \times\left[\mathbf{H}_{e}+\alpha \mathbf{n}_{\mathbf{m}} \times\left(\mathbf{H}_{e}-\mathbf{H}_{s}\right)\right]
$$

where we have used the Landau-Lifshitz-Gilbert equation including the spin torque term $\mathbf{H}_{s}=(I p)(\hbar / 2 q)(1 / \alpha m) \mathbf{n}_{s}$ ( $I$ is current density, $p$ is the spin polarization coefficient, $q$ is the electron charge, and $\mathbf{n}_{s}$ is a unit vector in the direction of the magnetization of the pinned layer). To make the solution mathematically tractable, we consider the case where the magnetic field $\mathbf{H}$ and $\mathbf{n}_{s}$ are parallel to the anisotropy axis, i.e., $\mathbf{H}_{e}=\left(H+H_{k} \cos \theta\right) \mathbf{e}_{z}$ and $\mathbf{n}_{s}=\mathbf{e}_{z}$ where $H_{k}=2 K / M_{s}$. With these simplifications, Eq. (3) reduces to

$$
\frac{\partial P}{\partial t}=\frac{\alpha \gamma}{m} \frac{\partial}{\partial x}\left[\left(1-x^{2}\right)\left(\frac{\partial U_{e}}{\partial x} P+k_{B} T \frac{\partial P}{\partial x}\right)\right]
$$

where $x=\cos \theta$, and we have defined the effective potential $U_{e}=\left(H_{s}-H\right) m x-\left(H_{k} / 2\right) m x^{2}$. Equation (4) can be solved via the method of separation of variables. Let's assume $P(x, t)=$ $f(t) u(x)$ and it is easy to see that $f(t)=\exp (-\lambda t)$ and $u(x)$ satisfies

$$
\frac{\alpha \gamma}{m} \frac{d}{d x}\left[\left(1-x^{2}\right)\left(\frac{d U_{e}}{d x} u(x)+k_{B} T \frac{d u(x)}{d x}\right)\right]=-\lambda u(x)
$$

The above equation can be converted into a Sturm-Liouville equation,

$$
\frac{d}{d x}\left[\left(1-x^{2}\right) \mathrm{e}^{-\beta U_{e}} \frac{d F}{d x}\right]+c \mathrm{e}^{-\beta U_{e}} F=0
$$

where $F(x)=\mathrm{e}^{\beta U_{e}(x)} u(x)$ and $c=\lambda m / \alpha \gamma k_{B} T$. Equation (6) is the same equation introduced by Brown [2] except that $U_{e}$ includes the spin-current term $H_{s}$. The original FPE (4) is 
now reduced to the standard eigenvalve problem. Namely, we determine eigen-function $F(x)=F_{n}(x)$ and eigenvalue $\lambda=\lambda_{n}$ from Eq. (6) for $n=0,1,2, \ldots$, and the general solution of Eq. (4) is

$$
P(x, t)=\sum_{n=0} A_{n} e^{-\beta U_{e}(x)} F_{n}(x) \mathrm{e}^{-\lambda_{n} t}
$$

where the coefficients in Eq. (7) are determined by the initial condition Eq. (1): $A_{n}=$ $\int_{-1}^{1} d x P(x, 0) F_{n}(x)$, where we have chosen the eigenfunction $F_{n}(x)$ to be weighted normalized: $\int_{-1}^{1} d x \mathrm{e}^{-\beta U_{e}(x)} F_{n}^{2}(x)=1$.

The remaining task is to solve eigenvalue $\lambda_{n}$ (or $c_{n}$ ) and eigenfunction $F_{n}$. Obviously, $\lambda_{0}=0$ is the lowest eigenvalue with the corresponding eigenfunction $F_{0}$ being a constant. Thus, $u_{0}(x) \propto \exp \left(-\beta U_{e}\right)$ describes the thermal equilibrium state. The smallest nonzero eigenvalue $\lambda_{1}$ then determines the slowest decaying or switching speed.

The early theoretical attempts toward solving Eq. (6) [2, 3, 4, 5, 6] had been focused on the cases where there are two potential wells separated by an energy barrier and $H<H_{k}$. These solutions do not apply to the fast switching case where $H>H_{k}$. In this case, there is no energy barrier and the only stable solution is at $x=-1$ or $\theta=\pi$. In the following, we develop two methods to solve the Eq. (6): one is the approximate minimization which provides an upper bound for $\lambda_{1}$ and the other is to numerically determine the coefficients of polynomial series when Eq. (6) is expanded into polynomials.

The variational method can be obtained by multiplying $F(x)$ to Eq.(6) and then by integrating from -1 to 1 ,

$$
\int_{-1}^{1} d x F \frac{d}{d x}\left[\left(1-x^{2}\right) \mathrm{e}^{-\beta U_{e}} \frac{d F}{d x}\right]+c \int_{-1}^{1} \mathrm{e}^{-\beta U_{e}} F^{2} d x=0 .
$$

Performing integration by parts of the first term, we find

$$
c=\mathcal{D} / \mathcal{H}
$$

where

$$
\left\{\begin{array}{l}
\mathcal{D}=\int_{-1}^{1} d x \mathrm{e}^{-\beta U_{e}}\left(1-x^{2}\right)\left(\frac{d F}{d x}\right)^{2} \\
\mathcal{H}=\int_{-1}^{1} d x \mathrm{e}^{-\beta U_{e}} F^{2}(x)
\end{array}\right.
$$

Since both $\mathcal{D}$ and $\mathcal{H}$ are definitely positive, the variational principle is to choose a trial function $F(x)$ such that $c$ is minimized, and the non-zero minimum $c$ represents the upper 
bound of the lowest eigenvalue $c_{1}$. Additional constriction of $F(x)$ is its orthogonality with $F_{0}$ which is a constant, i.e.,

$$
\int_{-1}^{1} \mathrm{e}^{-\beta U_{e}} F(x) d x=0 .
$$

Since $\exp \left(-\beta U_{e}\right)$ is sharply peaked at $x=-1$ in our case, we change the variable as $x=y-1$ and thus $\beta U_{e}=\eta y-\xi y^{2}+$ const, where we define $\eta=\frac{m}{k_{B} T}\left(H_{s}-H+H_{k}\right)$ and $\xi=\frac{m}{k_{B} T} \frac{1}{2} H_{k}$. We consider a simple trial function $F(y)=1+b_{1} y+b_{2} y^{2}$ where $b_{1}$ and $b_{2}$ are variational parameters. By placing $F(y)$ into Eq. (9) and (10), we have

$$
\left\{\begin{array}{l}
\mathcal{D}=\int_{0}^{2} y(2-y) \mathrm{e}^{-\eta y+\xi y^{2}}\left(b_{1}+2 b_{2} y\right)^{2} d y \\
\mathcal{H}=\int_{0}^{2} \mathrm{e}^{-\eta y+\xi y^{2}}\left(1+b_{1} y+b_{2} y^{2}\right)^{2} d y \\
\int_{0}^{2} \mathrm{e}^{-\eta y+\xi y^{2}}\left(1+b_{1} y+b_{2} y^{2}\right) d y=0
\end{array}\right.
$$

After the completion of these three integrations, the eigenvalue $c_{1}$ is obtained by minimizing $\mathcal{D} / \mathcal{H}$ with respect to $b_{2}$ or $b_{1}$. The above integrations can be analytically obtained if we we notice that $y$ is limited to $1 / \eta$ due to exponential $\mathrm{e}^{-\eta y}$ and thus the term $e^{\xi y^{2}}$ is small and can be expanded as: $\mathrm{e}^{\xi y^{2}}=1+\xi y^{2}+\frac{1}{2} \xi^{2} y^{4}$. By using $\int_{0}^{2} y^{n} \mathrm{e}^{-\eta y} d y \approx n ! / \eta^{n+1}$, for example, the third equation in Eq. (11) is

$$
\begin{aligned}
\frac{1}{\eta}+\frac{2 \xi}{\eta^{3}} & +\frac{12 \xi^{2}}{\eta^{5}}+\left(\frac{1}{\eta^{2}}+\frac{6 \xi}{\eta^{4}}+\frac{60 \xi^{2}}{\eta^{6}}\right) b_{1} \\
& +\left(\frac{2}{\eta^{3}}+\frac{24 \xi}{\eta^{5}}+\frac{360 \xi^{2}}{\eta^{7}}\right) b_{2}=0
\end{aligned}
$$

Note that the leading orders of $b_{1}$ and $b_{2}$ are $\eta$ and $\eta^{2}$ respectively, and the above equation is valid up to $\eta^{-3}$. If we consider the fast switching case, i.e. $\eta \gg \xi$, or $H_{s}-H \gg H_{k}$, the equation (12) can be further approximated as

$$
b_{1}=-2 b_{2} \eta^{-1}-\eta
$$

We can similarly calculate $\mathcal{H}$ and $\mathcal{D}$. Substituting $b_{1}$ by Eq. (13), the expression of $\mathcal{D} / \mathcal{H}$ contains only $b_{2}$. Then, we find $b_{2}$ by minimizing $\mathcal{D} / \mathcal{H}$

$$
\frac{d}{d b_{2}}\left(\frac{\mathcal{D}}{\mathcal{H}}\right)=0
$$


The results are:

$$
\left\{\begin{array}{l}
b_{2}=\frac{1}{2} \eta^{2}, b_{1}=-2 \eta \\
\mathcal{H}=\frac{1}{\eta}, \quad \mathcal{D}=4, \quad c_{1}=\frac{\mathcal{D}}{\mathcal{H}}=4 \eta
\end{array}\right.
$$

Rewriting in terms of original parameters, we find the slowest decaying rate is

$$
\lambda_{1}=4 \alpha \gamma\left(H_{s}-H\right)=4\left(I / I_{c}-1\right) \tau_{0}^{-1}
$$

where $\tau_{0}^{-1}=\frac{p \mu_{B}}{q m} I_{c}=\alpha \gamma\left(H+H_{k}\right)$.

Although the variational method is able to analytically obtain the upper bound for the rate of the relaxation, its accuracy is unknown. A more rigorous method is to expand $F(x)$ via Taylor series, i.e., $F(x)=\sum a_{n} x^{n}(n=0,1,2 \ldots)$. By substituting it into Eq. (6), we find the recurrence formula for coefficient $a_{n}$

$$
\begin{array}{r}
(n+2)(n+1) a_{n+2}-\zeta(n+1) a_{n+1} \\
+\left[c_{n}-n(n-1)+n(2 \xi-2)\right] a_{n} \\
+\zeta(n-1) a_{n-1}-2 \xi(n-2) a_{n-2}=0
\end{array}
$$

where $\zeta=\eta-2 \xi=\frac{m}{k_{B} T}\left(H_{s}-H\right)$. In the case of $\zeta=\xi=0$, the above equation reduces to a two-term recursion formula, and the solutions are the Legendre polynomials with $c_{n}=$ $n(n+1)$. This is the well-known solution for the thermal decay of a free particle [2]. In the case of $\zeta=0$, Eq. (16) reduces to a three-term recursion formula, and the continued fraction method can be used to obtain the smallest nonzero eigenvalue $c_{1}=2-\frac{4}{5} \xi+O\left(\xi^{2}\right)[3]$.

In our case where both $\zeta$ and $\xi$ are non-zero, above methods can not be used directly. One way to solve the problem is to numerically calculate Eq. (16) by simply keeping enough terms (i.e. up to a large number $N=300$ ) to ensure the convergence of $c_{1}$. In Fig. 1, we show that the relaxation rates as a function of temperature $\left(H_{k}\right.$ is fixed) for different $I / I_{c}(\geq 1)$. In Fig. 2, we show that the relaxation rate is linearly dependent of $I / I_{c}-1$ when $I / I_{c}>1.5$. By comparing with Eq. (15) derived from the variational approach, we find that to a good approximation the relaxation rate is a factor of two smaller, i.e.,

$$
\lambda_{1}=2\left(I / I_{c}-1\right) \tau_{0}^{-1}
$$




\section{COMPARISON WITH SUN'S MODEL}

Sun's model [1] assumes that thermally distributed initial magnetization states determine the distribution of switching time in case of $I>I_{c}$. In this model, the switching time at zero temperature is estimated as $\tau=\tau_{0}\left(I / I_{c}-1\right)^{-1} \ln (\pi / 2 \theta)$, where $\theta$ is the initial angle of magnetization whose distribution is given by Eq. (1). The corresponding distribution of switching time is calculated from the definition: $D(\tau) d \tau=-P(\theta) \sin \theta d \theta$. By defining the switching probability density $D(\tau) d \tau=-P\left(\mathbf{n}_{\mathbf{m}}, 0\right) \sin \theta d \theta$ and utilizing the above relation between $\tau$ and $\theta$, the probability of not being switched is

$$
E_{r}(t) \equiv 1-\int_{0}^{t} D(\tau) d \tau \simeq \frac{\pi^{2} \xi}{4} \exp \left(-\frac{2\left(I / I_{c}-1\right)}{\tau_{0}} t\right)
$$

where a long time limit is taken. The relaxation rate is identical with Eq. (17). This suggests the initial-condition randomization is the leading cause for switching time distribution in the limit of large $\xi$ and $I / I_{c} \gg 1$. Deviation occurs when $\xi$ is small, such as shown in Fig. 1, or when the current is near $I_{c}$, as in Fig. 2 .

This work is supported in part by IBM.

[1] J. Z. Sun et. al., Proc. SPIE Vol.5359, 445 (2004).

[2] W. F. Brown, Jr., Phys. Rev. 130, 1677 (1963).

[3] A. Aharoni, Phys. Rev. 177, 793 (1969).

[4] C. N. Scully et. al., Phys. Rev. B 45, 474 (1992).

[5] W. T. Coffey et. al., Phys. Rev. B 51, 15947 (1995);

[6] X. Wang et. al., J. Appl. Phys. 92, 2064 (2002)

[7] R. H. Koch et. al., Phys. Rev. Lett. 92, 088302 (2004)

[8] Z. Li et. al., Phys. Rev. B 69, 134416 (2004);

[9] D. M. Apalkov et. al., Phys. Rev. B 72, 180405(R) (2005) 


\section{Figure Caption}

FIG.1(Color online) Numerical results of the smallest nonzero eigenvalues of Eq. (16) for increasing $\xi$, in cases of different $I / I_{c} \cdot \tau_{0}^{-1}=\alpha \gamma\left(H+H_{k}\right)$.

FIG.2(Color online) Numerical results of the smallest nonzero eigenvalues of Eq. (16) for increasing $I / I_{c}-1$, where $\xi=80$. The red line is the fitting line: $\lambda_{1} \tau_{0}=2\left(I / I_{c}-1\right)$. 


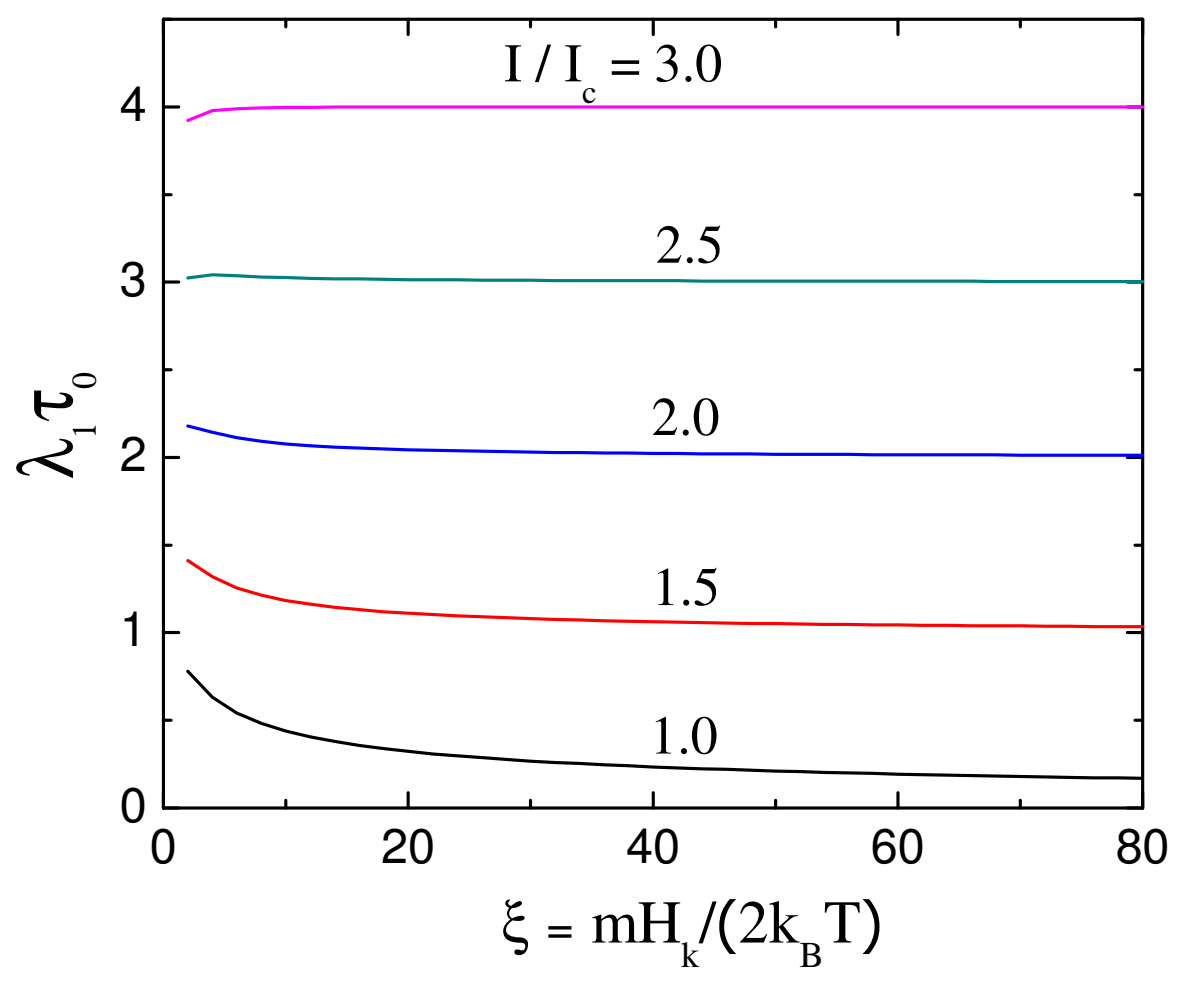

FIG. 1: 


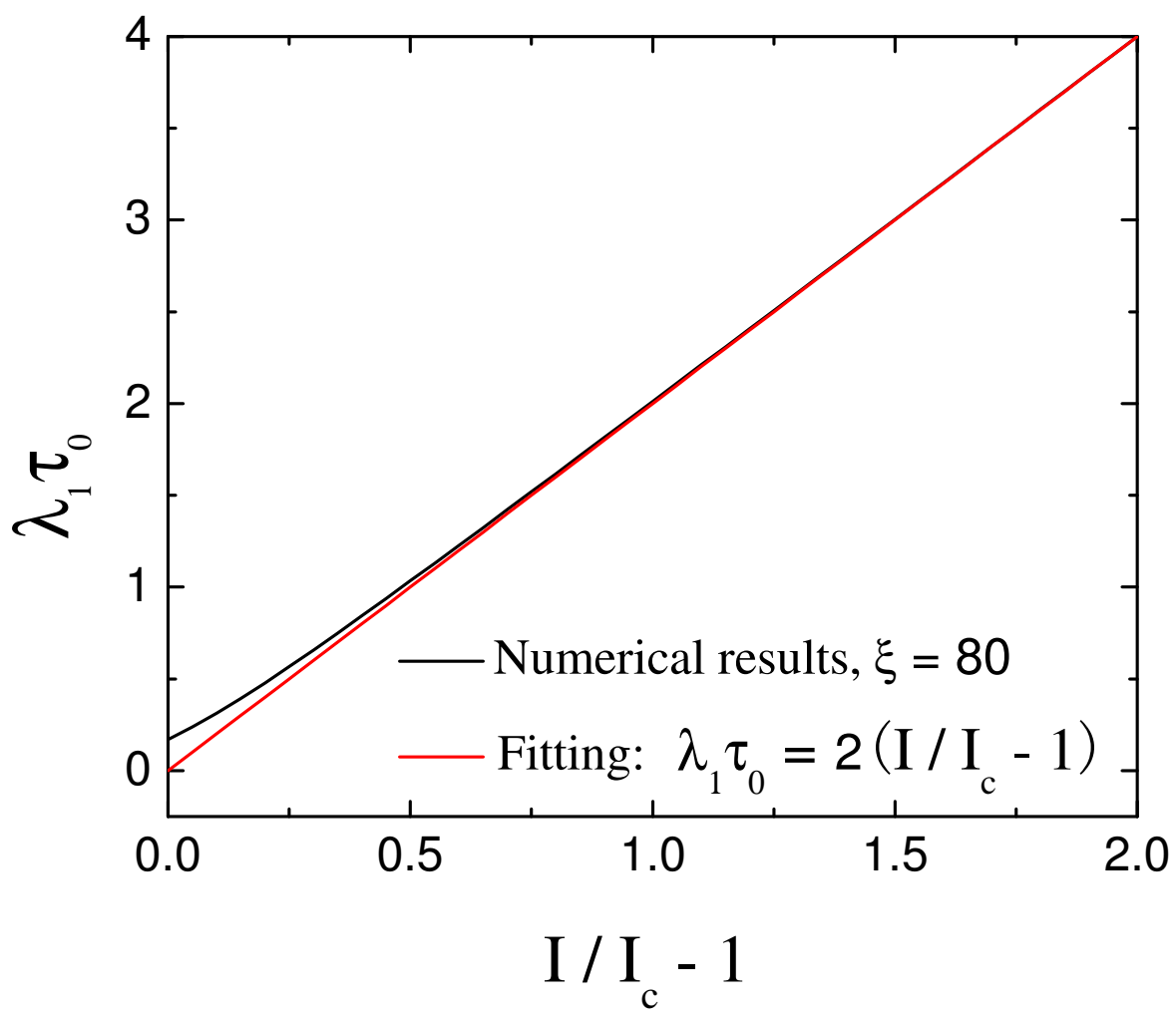

FIG. 2: 